Short report

\title{
Haemophilia Laboratory diagnosis training and care in Rural communities in Sudan
}

\author{
Fathelrahman M. Hassan ${ }^{1}$ and Maria M. Satti ${ }^{2}$ \\ ${ }^{1}$ College of Medical Laboratory Science, Sudan University of Science and technology, Khartoum, Sudan \\ ${ }^{2}$ Faculty of Medicine, Khartoum University, Khartoum, Sudan
}

Received 15 April 2012, Revised 2 May 2012, Accepted 23 May 2012

(C) 2012, Hassan F.M., Satti M.M.

(C) 2012, Russian Open Medical Journal

Abstract: Sixty nine per cent of people with hemophilia symptoms in rural areas were accessed to laboratory diagnosis and care support in Sudan, where technical expertise and health care facilities was less than optimal. There were many reasons for the inadequate care of hemophilic patients: the perception of rarity of the disease; lacked of laboratory facilities to diagnose the disorder; lacked of understanding of the disorder by patients, their relatives, and even healthcare providers; poorly developed blood bank facilities; and lacked of adequate factor supply were just some examples. The Sudanese Hemophilia Care Association (SHCA) was attempted to address many of these issues by establishing hemophilia care programs and by educating and training healthcare practitioners so that a healthcare team could be organized that attempts to ameliorate these problems and provides treatment options. However, it was possible to manage hemophiliac's patients with limited resources. Strategies for conserving factor concentrates were included education of doctors and patients, prenatal diagnosis, increasing the use of anti fibrinolytic agents, physiotherapy, the use of fibrin glue, and simple orthotics and prosthetic measures. An outreach program would be initiated to ensure that hemophilia care and diagnosis was available outside the capital city. Official recognition of hemophilia laboratory diagnosis and treatment centers and designated centers by the government could also be very beneficial in ensuring adequate care in rural areas in Sudan.

Keywords: hemophilia, laboratory diagnosis, rural communities

Cite as Hassan FM, Satti MM. Haemophilia Laboratory diagnosis training and care in Rural communities in Sudan. Russian Open Medical Journal 2012; 1: 0103.

Correspondence to Fathelrahman M. Hassan. Address: P.O.Box: 407. College of Medical Laboratory Science, Sudan University of Science and Technology, Khartoum, Sudan. Phone: +249912361571. E-mail: fathmaga@yahoo.com.

\section{Introduction}

There are just a few people in Sudan Rural community who know about Hemophilia. Hemophilia is a rare inherited disorder in which the blood does not clot normally [1]. Persons with Hemophilia have low blood clotting, if they bleed, it can't be stopped easily [2]. They need infusions of clotting factor regularly to maintain their health. If bleeding occurs frequently near joints and doesn't get a proper medication, the joints will be damaged and the patient will end up with a physical disability [3]. Hemophilia laboratory diagnosis and care is making progress in the large cities. However, up to $96 \%$ people with hemophilia symptoms in rural areas have access to laboratory diagnosis and care support. An urgent task is to extend regional hemophilia diagnosis and care into these areas. The government spending on health has been significantly reduced. These difficulties were reflected in the insufficient resources allocated to the sector and inefficiency in the utilization of the resources, unequal geographical distribution of the health care facilities and personnel, the deterioration in the work environment, and the continuous decline in the work force in the sector. However, the economic reforms of the liberalization policies have led to fundamental changes in the health sector [4]. Health care facilities have been negatively affected by the shortage of finance resulting from the curtailment of government spending allocated to health services and the limited success of the newly introduced co- payment system [4]. Based on the previous work this study will test the hypothesis that providing hemophilia laboratory diagnosis training and care in rural communities in Sudan will improve accessibility diagnosis and care for thousands of people with hemophilia symptoms in these areas.

The aim of this work was to promote and provide hemophilia laboratory diagnosis training and care in rural communities in Sudan, develop hemophilia laboratory diagnosis training and care Guide/curriculum and teaching materials appropriate for use in rural communities in Sudan and significantly improve hemophilia laboratory diagnosis and care support available to people with hemophilia in Sudan.

\section{Materials and Methods}

This study included 670 Sudan participants (442 males and 228 females) living in four Sudanese cities (Khartoum, Madani, Kosti and Senar). The Study groups were divided into three age groups: under 15 years, 15 to 64 years and equal to or above 65 years.

The hemophilia laboratory diagnosis working group were appointed a planning team for conducting laboratory diagnosis and care training at Kharoum, Madani, Kousti, and Singa regions, each course was included; lectures, seminars and a review session for medical laboratory technologists and hemophilic patients with their families. The feedback and experience gained from training 
courses was used by the planning team to prepare a training guide with a curriculum, lectures and teaching tools. These materials were prepared in many formats, including a printed manual, CD and video. The teaching tools were also available on the internet for easy access and to enable self-training. To improved skills of the laboratory technologists and patients care in the rural communities the training guide was set basic standards for training and providing a free kit of teaching materials to reduced costs, simplify organization and motivated more facilitators to providing training in the rural communities in Sudan. The project structure was included many activities in order to perform to promote the technical and scientific study's results and ensure the widest use of knowledge derived from the project. The plan - aimed at the potential interested parties in the rural communities as well as at participants of the project.

\section{Results}

Current situation of haemophilia care in Sudan was found to increase the under-diagnosed new cases and scattered fragmented care. Out of 670 participants; 442 were males and 228 were females, the mean age was 27.7 years and most patients age group (61.0\%) were between 15 to 64 years (Table 1 ).

\section{Table 1. Patients Characteristics}

\begin{tabular}{lc}
\hline Parameter & $\begin{array}{c}\text { All patients } \\
(\mathrm{n}=670)\end{array}$ \\
\hline Age, years, mean \pm SD & $27.7 \pm 3.2$ \\
Sex, no. (\%) & \\
- Male & $442(66.0 \%)$ \\
- Female & $228(34.0 \%)$ \\
Age groups, no. (\%): & \\
- Under 15 years & $175(26.0 \%)$ \\
- 15 - 64 years & $409(61.0 \%)$ \\
- $\geq 65$ years & $86(13.0 \%)$ \\
Life expectancy at birth, years: & \\
- Total populations & 72.0 \\
- Male & 66.3 \\
- Female & 69.2 \\
Sex Ratio (males/females): & \\
- Total population & 1.24 \\
- Under 15 years & 1.04 \\
- 15 - 64 years & 1.43 \\
- $\geq 65$ years & 1.18 \\
\hline
\end{tabular}

Table 2. The number of individuals who have benefitted (directly and indirectly) from Hemophilia Laboratory diagnosis training and care in Rural communities in Sudan

\begin{tabular}{lcc}
\hline & End User & $\begin{array}{c}\text { Other } \\
\text { Beneficiaries }\end{array}$ \\
\hline $\begin{array}{l}\text { Number in Khartoum Area } \\
\text { Number in Madani Area }\end{array}$ & 87 & 12 \\
Number in Senar Area & 64 & 14 \\
Number in Kosti Area & 19 & 1 \\
Number in educational settings & 37 & 8 \\
Number in health care settings & 41 & 9 \\
$\begin{array}{l}\text { Other end users/other (specify): Family } \\
\text { members located in distant states }\end{array}$ & 213 & 150 \\
$\begin{array}{l}\text { Total number of direct and indirect } \\
\text { beneficiaries }\end{array}$ & 15 & 0 \\
\hline
\end{tabular}

The life expectancy at birth for total population was 72 years with minor variation in male to female. Sex Ratio (males/females) was higher in the range of 15 to 64 years for up to 1.43 compared to other age groups as shown in Table 1 . Most of patients (60.0\%) were living in Khartoum state (Figure 1). The number of individuals who have benefitted (directly and indirectly) from Hemophilia Laboratory diagnosis training and care in rural communities; 476 of them were end user of the program and 194 of them were other Beneficiaries related to the program as shown in Table 2.

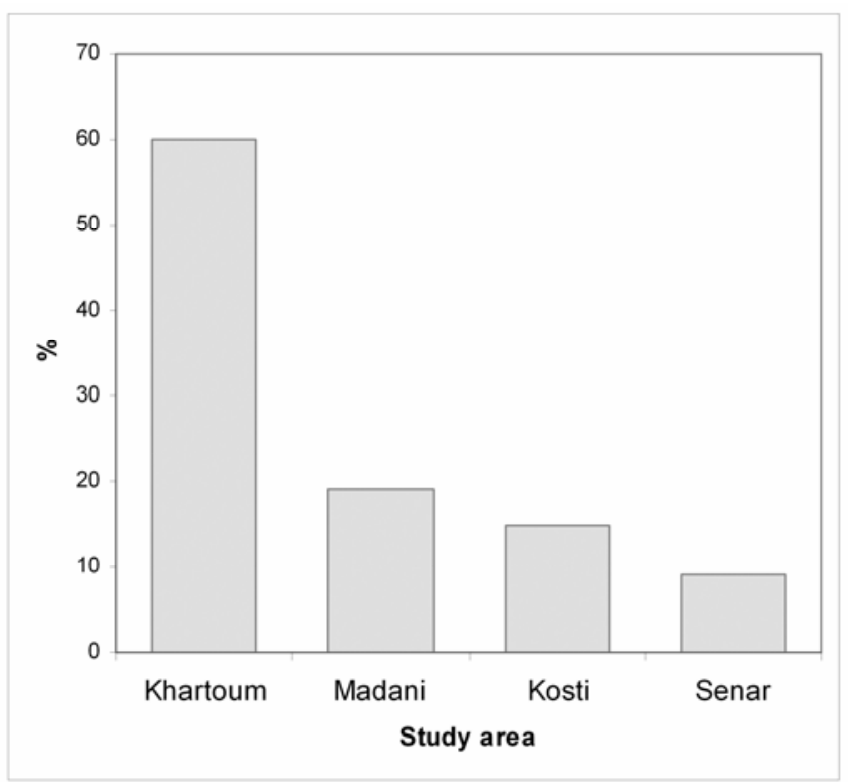

Figure 1. Current hemophilia distribution status in Sudan

\section{Discussion}

Providing haemophilia care in developing countries is a challenge. Caregivers not only have to walk a tight rope regarding the amounts of factor concentrates needed for individual patients, but must also be conversant with factor-concentrate requirements for individual patients and factor-concentrate saving techniques and devices. Patient education was another great challenge, needing careful implementation in countries where literacy rates were still low. Some centres in developing countries provide prenatal diagnosis of haemophilia [3], but this was too restricted and needs to be made available more widely.

Hemophilia laboratory diagnosis training and care Guide/curriculum was improved in rural communities in Sudan. To train the healthcare team, the WFH uses a range of medical training programs, such as the International Haemophilia Training Centre Fellowships [5] and the Centre Twinning Program [6], as well as laboratory workshops.

Hemophilia laboratory diagnosis and care would support people with hemophilia to realize the main problem in doing their activities. If they could get regular medication they can live healthy and productive lives. That's why we struggle hard to facilitate this group. Training and educational materials provided in this study would extend to prevention and patient education. Healthcare professionals had a significant role to play in educating patients and caregivers about how to minimize damage caused by hemophilia [7], and also, through genetic counseling, in helping patients, carriers, and their families make more informed choices about deciding to have children who could be affected by hemophilia. By approaching and creating awareness within the 
government and in insurance companies, all people with hemophilia in rural could now use a special healthcare insurance where medical expenses were covered by the government.

Through our hard efforts, our goal to enhance quality of life of people with hemophilia could become true. So they could contribute to the community of this country just like others did. Based on the results of the needs assessment, programs and services would be implemented in 2009 \& 2010. The team staff was worked with haemophilia committee to develop a first step program for new families and developed a fitness program for the bleeding disorders community.

Establishing of patient outreach was involved identifying individuals with hemophilia or other bleeding disorders who have not yet been diagnosed or whose health needs were not being met by healthcare services. Patients could be identified through awareness-raising and education activities or by actively seeking out patients for diagnosis. Patient outreach was focused on a targeted population, in this case people with hemophilia, von Willebrand disease or other hereditary bleeding disorder, with the aim of identifying them were provided them with proper treatment and healthcare services.

Finding potential patients and arranging for diagnosis was usually done by organizing a patient outreach campaign within a specific community, country, or region. It is hope that the established of the hemophilia laboratory diagnosis training and care will promote interest and awareness on hemophilia laboratory diagnosis and improve skills of the laboratory technologists in the rural communities. Like other children and youth, those with special health care needs like children with Hemophilia, deserves to be happy, had a healthy childhood, attended school, enjoyed community events, met with friends and neighbors and had the opportunity to become productive adults.

There were many reasons for the inadequate care of hemophilic patients: the perception of rarity of the disease; lacked of laboratory facilities to diagnose the disorder; lacked of understanding of the disorder by patients, their relatives, and even healthcare providers; poorly developed blood bank facilities; and lacked of adequate factor supplied were just some examples.

The WFH attempts to address many of these issues by establishing hemophilia care programs and by educating and training healthcare practitioners so that a healthcare team was organized that attempts to ameliorate these problems and provides treatment options. In the last few years, a considerable number of developing countries had been organized to deliver at least a minimum of care, and attempts had been made to obtain support from appropriate governmental sources.

Developing treatment guidelines and protocols based on the WFH models were help ensure an accepted standard for hemophilia care throughout a country. Given that less than $30 \%$ of patients with hemophilia were diagnosed, improving laboratory diagnosis was a key focus area. The WFH had developed a laboratory manual [8] and practical hands-on workshops to teach the basic diagnostic techniques which would be a guideline for laboratory diagnosis and care in rural community in Sudan.

\section{Conclusion}

Most patients with haemophilia were living in Khartoum state, so; the training and providing a free kit of teaching materials was useful in reduce costs, simplify organization and motivate more facilitators to provide training in the rural communities.
Information would be shared by valuable to develop working team for an appropriate training curriculum and materials to improve the quality of lives of individuals and families affected by bleeding disorders. Educating and training clinicians, laboratory technologists, and other healthcare professionals was essential to ensure adequate expertise in diagnosis and treatment.

Hemophilia treatment centers in Sudan would be more efficient and accessible on a national basis. This would included the setup of at least one (and preferably several) hemophilia treatment centers, designation of dedicated clinicians and a comprehensive care team with a specific interest in hemophilia, diagnosis by designated laboratories, and the compilation of a national registry in rural community.

An outreach program would be initiated to ensure that hemophilia care and diagnosis was available outside the capital city. Such other services were needed for comprehensive cares were; patient and family education, physiotherapy and subspecialties such as; Rheumatologist, Orthopaedic surgery, Hepatologists, Orthotics, Dentist and Infectious diseases specialist.

Replacement therapy was not always available because of high cost of replacement therapy which was needed for more health sectors commitment. The lack of medical staff \& technologist training and multiple health care systems results for an inadequate care: rare (not priority for health care planner), Inadequate infrastructure for diagnosis, poorly developed blood transfusion services and high cost of factors.

\section{Conflict of interest}

No conflict of interest.

\section{Acknowledgements}

We thank the Bayer hemophilia award foundation for caregiver award to support this project.

\section{Reference}

1. Kessler CM. Hemorrhagic disorders: coagulation factor deficiencies. In: Goldman L, Ausiello D, eds. Cecil Medicine. 23rd ed. Philadelphia, Pa: Saunders Elsevier; 2007:chap. 180.

2. Kessler CM, Tfayli A. Coagulation disorders: Acquired and congenital. In: B Furie et al., eds., Clinical Hematology and Oncology, chap. 58, pp. 498-510. Philadelphia: Churchill Livingstone, 2003.

3. Ghosh K, Shetty S, Pawar A, Mohanty D. Carrier detection and prenatal diagnosis in haemophilia in India. Realities and challenges. Haemophilia 2002; 8(1): 51-55 (PMID: 11886465) (doi: 10.1046/j.1365-2516.2002.00576.x).

4. Regional Health Systems Observatory - EMRO, Health System ProfileSudan, Health Expenditure Data and Trends, 2005: 27-28.

5. Rickard K. The International Hemophilia Training Centres of the World Federation of Hemophilia: a 30-year review. Haemophilia 2000; 6(5): 471-473 (PMID: 11012687) (doi: 10.1046/j.1365-2516.2000.00446.x).

6. Improving Care Beyond Our Borders: A Twinning Guide for Hemophilia Treatment Centres. Montreal: WFH; 2002.

7. Haemophilia in Pictures. Montreal: WFH; 1996.

8. Diagnosis of Haemophilia and Other Bleeding Disorders: A Laboratory Manual. Montreal: WFH; 2000.

Authors:

Fathelrahman M. Hassan - College of Medical Laboratory Science, Sudan University of Science and technology, Khartoum, Sudan;

Maria M. Satti - Faculty of Medicine, Khartoum University, Khartoum, Sudan. 